\title{
Absolutely stable proton and lowering the gauge unification scale
}

\section{S.M. Barr ${ }^{a}$ and X. Calmet ${ }^{b}$}

${ }^{a}$ Department of Physics and Astronomy, Bartol Research Institute, University of Delaware, Newark, Delaware 19716, U.S.A.

${ }^{b}$ Physics \& Astronomy, University of Sussex, Falmer, Brighton, BN1 9QH, U.K.

E-mail: smbarr@bartol.udel.edu, x.calmet@sussex.ac.uk

ABstract: A unified model is constructed, based on flipped $\mathrm{SU}(5)$ in which the proton is absolutely stable. The model requires the existence of new leptons with masses of order the weak scale. The possibility that the unification scale could be extremely low is discussed.

Keywords: GUT, Beyond Standard Model, Rare Decays, Neutrino Physics

ARXiv EPRINT: 1404.4594 


\section{Contents}

1 Introduction 1

2 A unified model with an absolutely stable proton 2

3 Proof of proton stability and gauging "baryon number" $\quad 6$

4 Anomaly-freedom, and possible embedding in $\mathrm{SU}(6) \times \mathrm{SU}(2) \quad 7$

5 The lepton phenomenology of the model 9

$\begin{array}{lll}6 & \text { Low scale grand unification? } & 11\end{array}$

\section{Introduction}

It is an interesting question how low the unification scale can be. The most obvious issue is the proton lifetime. This is not necessarily a severe constraint on models of quarklepton unification based on the Pati-Salam group [1], since the gauge interactions in such models do not violate baryon number. And recently the possibility of a very low scale for Pati-Salam unification of quarks and leptons has been discussed [2].

Proton decay is a much more serious constraint on models with gauge unification, by which we mean the unification of $\mathrm{SU}(3)$ of color and $\mathrm{SU}(2)$ of weak isospin in a single simple gauge group. (Gauge unification includes both "grand" unification and "flipped" unification [3].) A very low gauge unification scale would be conceivable, however, if somehow the proton could be rendered absolutely stable. Of course, the lower the unification scale the less room for the Standard Model gauge couplings to run. Nevertheless, if there were significant corrections to the gauge couplings coming from new physics at or just above the unification scale, gauge coupling unification might nevertheless occur with a relatively low unification scale, perhaps even quite near the weak scale.

In section 2 of the paper, we construct a simple model based on flipped SU(5) [4-6] in which the proton is absolutely stable due to an exact local symmetry. We prove the absolute stability of the proton in the model in section 3 . In section 4 , we show that the symmetry that forbids proton decay in the model is anomaly-free and can therefore be gauged, thus ensuring that quantum gravity effects respect it. We also show that the model can be embedded in $\mathrm{SU}(6) \times \mathrm{SU}(2)$.

In section 5, we discuss the lepton sector, which has a rich low-energy phenomenology. The model requires the existence of several extra lepton doublets whose masses come from the Standard Model Higgs field and which therefore should be accessible to accelerator searches. Such extra lepton doublets would have a large effect on the amplitude for Higgs 
decay to two photons. We show how agreement with experiment is nonetheless possible. (The $H \rightarrow \gamma \gamma$ decay rate can also be smaller or larger than the Standard Model prediction, depending on the particle content of the model.) In section 5, we also show that small Majorana masses for the neutrinos can arise in a simple way despite the absence of superheavy right-handed neutrinos in the model.

In section 6 , we briefly discuss the unification of gauge couplings, and consider the radical possibility that the unification scale could be near the weak scale, and even more radically that the gravity scale could be near the weak scale as well, eliminating all large mass hierarchies.

\section{A unified model with an absolutely stable proton}

Proton decay can be suppressed if some of the baryon-number-violating gauge and Higgs couplings to quarks and leptons have the effect of converting $u$ or $d$ quarks into fermions that are too heavy to appear in the final state of proton decay. One could call this "kinematic blocking" of proton decay.

The most obvious way this might happen is that the unified interactions convert fermions of the lighter families into those of the heavier families, for example a $d$ quark into a $\tau$ lepton. If the unified group is $\mathrm{SU}(5)$ and only the Standard Model quarks and leptons exist, then proton decay cannot be completely suppressed by kinematic blocking even at tree-level, as it was shown already in [7-9]. On the other hand, it was shown in [10] that it can be completely suppressed at tree-level if the group is flipped $\mathrm{SU}(5)$, but only by tuning certain fermion mixing angle within SU(5) multiplets.

If non-Standard Model quarks and leptons are introduced, more possibilities exist for kinematic blocking of proton decay. Already in 1980 an SU(5) model in which proton decay was kinematically blocked by heavy non-Standard Model fermions was proposed in [11]. That model, however, is no longer viable, as the new quarks and leptons it proposed should have been seen by experiments. In another interesting paper [12], it was shown that proton decay can be forbidden to all orders in perturbation theory in $\mathrm{SU}(5)$ by introducing into each family extra vectorlike fermions in $\mathbf{5}+\overline{\mathbf{5}}+2 \times(\mathbf{1 0}+\overline{\mathbf{1 0}})$. However, proton stability was due to global symmetries in that model, and thus not immune to gravitational and other non-perturbative effects. Moreover, the pattern of vacuum expectation values assumed in that model cannot be exact without fine-tuning. (Another interesting model with kinematic blocking of proton decay in a supersymmetric SU(5) model with extra dimensions is [13].)

Recently, in [14], it was shown that in flipped SU(5) with new non-Standard Model fermions complete kinematic blocking of proton decay can be achieved at tree level in a simple way without fine-tuning tuning of parameters. But even in that scheme, the proton decay was not absolutely forbidden, since loop-diagrams and non-perturbative effects could induce it.

The model we present here, which like that of [14] is based on flipped SU(5), has an absolutely stable proton. Flipped SU(5) seems to be the smallest unification group that allows proton decay to be completely forbidden by kinematic blocking in a realistic model without any fine tuning of mixing angles. In this model, as in [14], the new types of heavy 


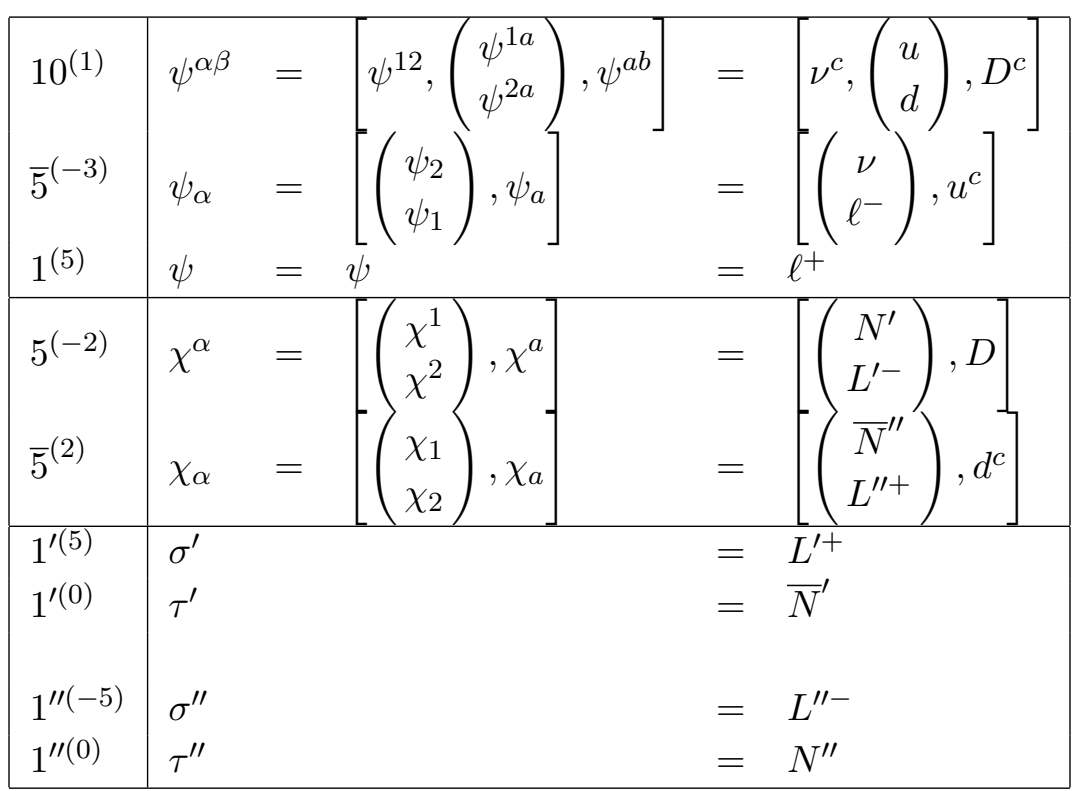

Table 1. The fermion content of one family of the model. The left column gives the $\mathrm{SU}(5) \times \mathrm{U}(1)_{X}$ quantum numbers. The other column shows how the different species of quark and lepton are contained in the SU(5) multiplets.

fermions that block proton decay are quarks and leptons that are vectorlike under the Standard Model group. These vectorlike fermions will be assumed to have masses of at least hundreds of GeV. We will denote these new heavy fermions by capital letters, and the known Standard Model fermions by lower-case letters.

The mechanism for blocking proton decay does not depend on the number of families, so we will suppress family indices throughout this paper for notational simplicity; and the discussion will proceed just as though there were only one family. It will be obvious that this does not matter.

The gauge group of "flipped $\mathrm{SU}(5)$ " is $\mathrm{SU}(5) \times \mathrm{U}(1)_{X}$, with the weak hypercharge $Y / 2$ of the Standard Model being a linear combination of the $\mathrm{U}(1)_{X}$ generator $X$ and the $\mathrm{SU}(5)$ generator $Y_{5} / 2 \equiv \operatorname{diag}\left(\frac{1}{2}, \frac{1}{2},-\frac{1}{3},-\frac{1}{3},-\frac{1}{3}\right)$, specifically $Y / 2=\frac{1}{5}\left(-Y_{5} / 2+X\right)$, with $X$ normalized as in table 1 , which gives the quark and lepton content of the model. The left column in table 1 gives the $\mathrm{SU}(5) \times \mathrm{U}(1)_{X}$ representation, where the superscript is the value of $X$. In the rest of the table, the Greek indices run from 1 to 5 and are the fundamental indices of $\mathrm{SU}(5)$. The index values 1,2 correspond to the weak hypercharge group $\mathrm{SU}(2)_{L}$, while the values $3,4,5$ correspond to the $\mathrm{QCD}$ color group $\mathrm{SU}(3)_{c}$ and are denoted by lower-case Latin letters, $a, b$, etc. (Note, however, that the superscript $c$ when appearing alone denotes an antiparticle, for example $d^{c}$ denotes the anti-down quark.)

One sees from table 1 that a single family of fermions in this model consists of the usual $10^{(1)}+\overline{5}^{(-3)}+1^{(5)}$ family of flipped SU(5) (denoted by the letter $\psi$ ), plus a vectorlike pair $5^{(-2)}+\overline{5}^{(2)}$ (denoted by $\chi$ ), and a vectorlike set of SU(5)-singlet fermions, denoted by $\sigma$ and $\tau$. This may look like a somewhat arbitrary assortment, but, as will be seen in section 4 , they fit exactly into a small set of representations of $\mathrm{SU}(6) \times \mathrm{SU}(2)$, namely $(15,1)+$ 


\begin{tabular}{|l|ll}
\hline $5_{H}^{(-2)} H^{\alpha}=\left[\left(\begin{array}{c}H^{1} \\
H^{2}\end{array}\right), H^{a}\right]$ & $=\left[\left(\begin{array}{c}H^{0} \\
H^{-}\end{array}\right), H^{-1 / 3}\right]$ \\
$5_{H}^{(3)} \tilde{H}^{\alpha}=\left[\left(\begin{array}{c}\tilde{H}^{1} \\
\tilde{H}^{2}\end{array}\right), \tilde{H}^{a}\right]$ & $=\left[\left(\begin{array}{c}\tilde{H}^{+} \\
\tilde{H}^{0}\end{array}\right), \tilde{H}^{2 / 3}\right]$ \\
$10_{H}^{(1)} \Omega^{\alpha \beta}=\left[\Omega^{12},\left(\begin{array}{c}\Omega^{1 a} \\
\Omega^{2 a}\end{array}\right), \Omega^{a b}\right]=\left[\begin{array}{c}\left.\Omega^{0},\left(\begin{array}{c}\Omega^{2 / 3} \\
\Omega^{-1 / 3}\end{array}\right), \Omega^{-2 / 3}\right]\end{array}\right.$
\end{tabular}

Table 2. The Higgs field content of the model.

$(\overline{6}, 2)+(1,3)$. (In fact, the smallest anomaly free chiral set of fermions of $\mathrm{SU}(6) \times \mathrm{SU}(2)$ would consist of $(15,1)+(\overline{6}, 2)$, so from the viewpoint of this larger group the fermions of this model form quite an economical set.)

Notice one peculiarity of the normal flipped SU(5) family shown in the first three lines of table 1: it contains all the fermions of a Standard Model family with the exception of $d^{c}$. Where the $d^{c}$ would normally be, one finds the new heavy field $D^{c}$. And, conversely, $d^{c}$ occupies the place where one would expect to find $D^{c}$ in the extra vectorlike pair $5^{(-2)}+\overline{5}^{(2)}$. This substitution is essentially what leads to the kinematic blocking of proton decay. How it happens will be seen shortly.

This explains the need for the extra vectorlike $5^{(-2)}+5^{(2)}$ of each family. What, however, explains the need for the extra SU(5)-singlet fermions (the ones denoted by $\sigma$ and $\tau)$ ? The answer is that they are there to "mate" with the leptons in $5^{(-2)}+\overline{5}^{(2)}$ to give them Dirac masses. One might ask whether these leptons could acquire mass more simply through a mass term $M \overline{5}^{(2)} 5^{(-2)}=M \chi^{\alpha} \chi_{\alpha}$. Indeed, they could; but such a term would necessarily include the term $M d^{c} D$, which would cause mixing between $d^{c}$ and $D^{c}$. That would mean that what we call $D^{c}$ in table 1 would, through mixing, actually be partly the light Standard Model field. This would cause the kinematic blocking of proton decay to be imperfect; and proton decay would only be suppressed by a mixing angle. This is what happens in the scheme proposed in [14]. This would not suppress proton decay sufficiently if the unification scale were very low. This is the reason why this scheme requires the existence of extra leptons whose masses come from electroweak breaking. These leptons have many phenomenological consequences, as will be discussed in section 5 . On the other hand, note that the extra quarks, $D$ and $D^{c}$, acquire mass from a Higgs field that is an electroweak singlet, i.e. $\Omega^{12}$, and do not couple to the Standard Model Higgs doublet. This difference between the extra quarks and extra leptons is a peculiar feature of the kind of model we are discussing.

Breaking the gauge symmetries and giving quarks and leptons masses can be done with just the three types of Higgs fields shown in table 2. As is usual in flipped SU(5) models, the breaking of $\mathrm{SU}(5) \times \mathrm{U}(1)_{X}$ down to the Standard Model group is done by a Higgs field that transforms as $10^{(1)}$, which we denote $\Omega^{\alpha \beta}$. The component $\Omega^{12}$ has $Y_{5} / 2=1$ and $X=1$ and so has $Y / 2=0$, and is also obviously a singlet under $\mathrm{SU}(3)_{c} \times \mathrm{SU}(2)_{L}$, so that 


\begin{tabular}{|llll|}
\hline$d d^{c}\left\langle\tilde{H}^{*}\right\rangle$ & $=\psi^{2 a} \chi_{a}\left\langle\tilde{H}_{2}^{*}\right\rangle$ & $\subset$ & $10^{(1)} \overline{5}^{(2)}\left\langle\left(5_{H}^{(3)}\right)^{*}\right\rangle$ \\
$\left(u u^{c}, \nu^{c} \nu\right)\left\langle H^{*}\right\rangle$ & $=\left(\psi^{1 a} \psi_{a}, \psi^{12} \psi_{2}\right)\left\langle H_{1}^{*}\right\rangle$ & $\subset$ & $10^{(1)} \overline{5}^{(-3)}\left\langle\left(5_{H}^{(-2)}\right)^{*}\right\rangle$ \\
$\ell \ell^{c}\langle H\rangle$ & $=\psi_{1} \psi\left\langle H^{1}\right\rangle$ & $\subset$ & $\overline{5}^{(-3)} 1^{(5)}\left\langle 5_{H}^{(-2)}\right\rangle$ \\
\hline$D^{c} D\langle\Omega\rangle$ & $=\psi^{a b} \chi^{d}\left\langle\Omega^{12}\right\rangle \epsilon_{12 a b d}$ & $\subset$ & $10^{(1)} 5^{(-2)}\left\langle 10_{H}^{(1)}\right\rangle$ \\
$L^{\prime-} L^{\prime+}\left\langle\tilde{H}^{*}\right\rangle$ & $=\chi^{2} \psi\left\langle\tilde{H}_{2}^{*}\right\rangle$ & $\subset$ & $5^{(-2)} 1^{\prime(5)}\left\langle\left(5_{H}^{(3)}\right)^{*}\right\rangle$ \\
$N^{\prime} \bar{N}^{\prime}\left\langle H^{*}\right\rangle$ & $=\chi^{1} \tau^{\prime}\left\langle H_{1}^{*}\right\rangle$ & $\subset$ & $5^{(-2)} 1^{\prime(0)}\left\langle\left(5_{H}^{(-2)}\right)^{*}\right\rangle$ \\
$L^{\prime \prime} L^{\prime \prime}\langle\tilde{H}\rangle$ & $=\sigma^{\prime \prime} \chi_{2}\left\langle\tilde{H}^{2}\right\rangle$ & $\subset$ & $1^{\prime \prime(-5)} \overline{5}^{(2)}\left\langle 5_{H}^{(3)}\right\rangle$ \\
$N^{\prime \prime} \bar{N}^{\prime \prime}\langle H\rangle$ & $=\tau^{\prime \prime} \chi_{1}\left\langle H^{1}\right\rangle$ & & \\
\hline$\Omega \tilde{H}^{*} H^{*}$ & $=\Omega^{12} \tilde{H}_{2}^{*} H_{1}^{*}$ & $\subset$ & $1^{\prime \prime(0)} \overline{5}^{(2)}\left\langle 5_{H}^{(-2)}\right\rangle$ \\
\hline$\nu N^{\prime \prime}\langle\tilde{H}\rangle$ & $=\psi_{2} \tau^{\prime \prime}\left\langle\tilde{H}^{2}\right\rangle$ & $\subset$ & $\overline{5}^{(-3)} 1^{\prime \prime(0)}\left\langle 5_{H}^{(3)}\right\rangle$ \\
\hline
\end{tabular}

Table 3. The Yukawa terms needed to give mass to the fermions. The ninth row is a term needed in the Higgs potential to align vacuum expectation values.

its vacuum expectation value (VEV) leaves the Standard Model group unbroken. This VEV is of order the unification scale. The Higgs fields that do the electroweak breaking are the $\mathrm{SU}(2)_{L}$ doublets in the $5_{H}^{(-2)}$ and $5_{H}^{(3)}$, which we denote respectively by $H$ and $\tilde{H}$.

There are eight Yukawa terms that are needed to give quarks and leptons mass, which are listed in the first seven rows of table 3, where we write these terms using the various alternative notations given in table 1 . The ninth row of table 3 gives a cubic term in the Higgs potential that is needed to tie the various Higgs fields together, thereby aligning their VEVs and avoiding accidental global symmetries in the Higgs potential that would lead to goldstone bosons.

The terms in the first nine rows of table 3 are needed in the model. There are also terms that must be forbidden if proton decay is to be suppressed. We already mentioned one such term, namely an explicit mass term of the form $\overline{5}^{(2)} 5^{(-2)}$, which would mix $d^{c}$ and $D^{c}$. Let us suppose for a moment that the terms in the first nine rows of table 3 are the only non-trivial terms in the Yukawa sector and Higgs potential. (We count as "trivial" any terms that always must be present no matter what the symmetries of the model are, such as the absolute square of any Higgs field.) With only those nine terms, the model is easily found to have a $\mathrm{U}(1) \times \mathrm{U}(1)$ accidental symmetry, which we will call $\mathrm{U}(1)_{a} \times \mathrm{U}(1)_{b}$.

In table 4 we give the $a$ and $b$ charges for all the fermion and Higgs multiplets listed in tables I and II. These charge assignments may look somewhat random, but it will be seen later that they have simple group theory interpretations if the flipped SU(5) group is embedded in $\mathrm{SU}(6) \times \mathrm{SU}(2)$. Moreover, note that the $b$ values have a simple pattern: fermions that are odd-rank $\mathrm{SU}(5)$ tensors have $b=-1$, those that are even-rank tensors 


\begin{tabular}{|c|ccc|cc|cccc|ccc|}
\hline field & $10^{(1)}$ & $\overline{5}^{(-3)}$ & $1^{(5)}$ & $5^{(-2)}$ & $\overline{5}^{(2)}$ & $1^{\prime(5)}$ & $1^{\prime(0)}$ & $1^{\prime \prime(-5)}$ & $1^{\prime \prime(0)}$ & $5_{H}^{(-2)}$ & $5_{H}^{(3)}$ & $10_{H}^{(1)}$ \\
$a$ & 0 & -1 & 2 & 0 & 1 & 1 & -1 & -2 & 0 & -1 & 1 & 0 \\
$b$ & 1 & -1 & 1 & -1 & -1 & 1 & 1 & 1 & 1 & 0 & 0 & 0 \\
\hline
\end{tabular}

Table 4. The $\mathrm{U}(1)_{a}$ and $\mathrm{U}(1)_{B}$ charges of the fields of the model.

have $b=+1$, and Higgs bosons have $b=0$. The $\mathrm{U}(1)_{a} \times \mathrm{U}(1)_{b}$ symmetry allows one more Yukawa interaction, $\overline{5}^{(-3)} 1^{\prime \prime(0)}\left\langle 5_{H}^{(3)}\right\rangle$, which is shown in the last row of table 3 . This term, which couples $\nu$ to $N^{\prime \prime}$, has the effect of mixing of $\nu$ and $\bar{N}^{\prime \prime}$. As we shall see, this is harmless and does not destabilize the proton. We will now prove that the symmetry $\mathrm{U}(1)_{a}$ forbids all operators of any dimension that would give proton decay.

\section{Proof of proton stability and gauging "baryon number"}

Any effective operator that leads to proton decay must involve only quark and lepton fields that are lighter than the proton, and thus not the new vectorlike fields denoted by capital letters in table 1. Consequently, it can be written in the general form

$$
\begin{aligned}
&(u, d)^{m}\left(u^{c}\right)^{n}\left(d^{c}\right)^{p}\left(\nu, \ell^{-}\right)^{q}\left(\ell^{+}\right)^{r}\left(\bar{N}^{\prime \prime}\right)^{s}\left(\left\langle H^{0}\right\rangle\right)^{t}\left(\left\langle\tilde{H}^{0}\right\rangle\right)^{u}(\langle\Omega\rangle)^{v} \\
& \subset\left(10^{(1)}\right)^{m}\left(\overline{5}^{(-3)}\right)^{n}\left(\overline{5}^{(2)}\right)^{p}\left(\overline{5}^{(-3)}\right)^{q}\left(1^{(5)}\right)^{r}\left(\overline{5}^{(2)}\right)^{s}\left(5_{H}^{(-2)}\right)^{t}\left(5_{H}^{(3)}\right)^{u}\left(10_{H}^{(1)}\right)^{v},
\end{aligned}
$$

where the exponents $m, n, p, q, r, s, t, u, v$ are integers. Note that here $d$ stands for either $d$ or $s$, and $e$ stands for either $e$ or $\mu$, since we are not showing family indices. Also note that we have included $\bar{N}^{\prime \prime}$ in this product. The reason is that this is not a purely heavy field, since (as we noted previously) there is mixing between the fields that are called $\nu$ and $\bar{N}^{\prime \prime}$ in table 1 , due to the last term in table 4 .

If $\mathrm{U}(1)_{a}$ is not explicitly broken, then the value of the generator $a$ of the operator in eq. (1) must vanish, giving

$$
-n+p-q+2 r+s-t+u=0 .
$$

By conservation of weak hypercharge, the value of $Y / 2$ of the operator in eq. (1) must vanish also, giving

$$
\frac{1}{6} m-\frac{2}{3} n+\frac{1}{3} p-\frac{1}{2} q+r+\frac{1}{2} s-\frac{1}{2} t+\frac{1}{2} u=0 .
$$

Multiplying eq. (3) by 2 and subtracting eq. (1) gives

$$
\frac{1}{3}(m-n-p)=B=0,
$$

where $B$ is baryon number. So no baryon-number-violating operators involving only quarks and leptons lighter than the proton exist to any order.

Note that the above arithmetic shows that the linear combination of generators

$$
\tilde{B} \equiv 2\left(\frac{Y}{2}\right)-a
$$


is the same as baryon number for the Standard Model fermions. (On the heavy new fermions, however, $\tilde{B}$ has peculiar values: the heavy quarks $D$ have $\tilde{B}=\frac{2}{3}$, and the heavy leptons $N^{\prime}$ and $L^{\prime}-$ have $\tilde{B}=-1$, with the corresponding antiparticles having the opposite values.) As we shall see in the next section, $\mathrm{U}(1)_{a}$ is anomaly free, and must be gauged in order to protect the stability of the proton from quantum gravity effects. Thus, in this model we are gauging a quantum number that coincides with baryon number on the Standard Model fields. The gauging of baryon number has been discussed in other recent papers $[15,16]$.

\section{Anomaly-freedom, and possible embedding in $\mathrm{SU}(6) \times \mathrm{SU}(2)$}

The proof of proton stability just given assumed that the symmetry $\mathrm{U}(1)_{a}$ is not explicitly broken. If it is a global symmetry, however, one would expect gravitational effects to break it explicitly. Therefore, to render the proton absolutely stable, it is necessary to gauge $\mathrm{U}(1)_{a}$, which would require that $\mathrm{U}(1)_{a}$ be anomaly-free. And indeed, it turns out that it is. With the set of fermions given in table 1 , and the $\mathrm{U}(1)_{a}$ charges given in table 4 , both the anomalies of $\mathrm{U}(1)_{a}$ alone and its mixed anomalies involving $\mathrm{SU}(5) \times \mathrm{U}(1)_{X}$ vanish. That is, the following five conditions are satisfied: $\operatorname{Tr}\left(a^{3}\right)=0, \operatorname{Tr}(a)=0, \operatorname{Tr}\left(a^{2} X\right)=0$, $\operatorname{Tr}\left(a X^{2}\right)=0$, and $\operatorname{Tr}\left(a\left(\lambda_{5}\right)^{2}\right)=0$ (where $\lambda_{5}$ is an $\mathrm{SU}(5)$ generator), as can easily be checked.

The satisfying of all these conditions seems like an amazing coincidence, but actually it has a simple explanation based on the group $\mathrm{SU}(6) \times \mathrm{SU}(2)$. The explanation consists in these three facts: (1) the group $\mathrm{SU}(6) \times \mathrm{SU}(2)$ contains $\mathrm{SU}(5) \times \mathrm{U}(1)_{X} \times \mathrm{U}(1)_{a}$; (2) the set of $\mathrm{SU}(6) \times \mathrm{SU}(2)$ representations $(15,1)+(\overline{6}, 2)+(1,3)$ is anomaly-free; and (3) when decomposed under $\mathrm{SU}(5) \times \mathrm{U}(1)_{X} \times \mathrm{U}(1)_{a}$ this set of representations contains exactly the set of fermions of one family of our model. We will now demonstrate each of these points.

That the set of $\mathrm{SU}(6) \times \mathrm{SU}(2)$ representations $(15,1)+(\overline{6}, 2)$ is anomaly-free is wellknown and follows simply from the possibility of embedding in $E_{6}$. That $(1,3)$ is anomalyfree follows simply from the fact that it is a real representation. Therefore, obviously, the combined set $(15,1)+(\overline{6}, 2)+(1,3)$ is anomaly-free under $\mathrm{SU}(6) \times \mathrm{SU}(2)$. Moreover, $\mathrm{SU}(6) \times \mathrm{SU}(2)$ obviously contains the subgroup $\mathrm{SU}(5) \times \mathrm{U}(1)_{6} \times \mathrm{U}(1)_{2}$, where $\mathrm{U}(1)_{6}$ is the subgroup of $\mathrm{SU}(6)$ corresponding to the diagonal generator $T_{6} \equiv \operatorname{diag}\left(\frac{1}{2}, \frac{1}{2}, \frac{1}{2}, \frac{1}{2}, \frac{1}{2},-\frac{5}{2}\right)$, and $\mathrm{U}(1)_{2}$ is the subgroup of $\mathrm{SU}(2)$ corresponding to the diagonal generator $T_{2} \equiv \operatorname{diag}\left(\frac{1}{2},-\frac{1}{2}\right)$. If one defines $X \equiv T_{6}+5 T_{2}$, then the $(15,1)+(\overline{6}, 2)+(1,3)$ is easily seen to decompose under $\mathrm{SU}(5) \times \mathrm{U}(1)_{X}$ into exactly the set of fermions in table 1 . And if one identifies $a$ with $2 T_{2}$, one immediately finds that those fermions have exactly the values of $a$ given in table 4 .

It should be mentioned that if the model is embedded in $\mathrm{SU}(6) \times \mathrm{SU}(2)$, then all the Yukawa couplings shown in table 3 can arise from just a few types of terms, namely terms of the form $(15,1)(15,1)\left\langle(15,1)_{H}\right\rangle,(15,1)(\overline{6}, 2)\left\langle(\overline{6}, 2)_{H}\right\rangle$, and $(\overline{6}, 2)_{H}(1,3)\left\langle(\overline{6}, 2)_{H}\right\rangle^{*}$.

The symmetry $\mathrm{U}(1)_{b}$ is not contained in $\mathrm{SU}(6) \times \mathrm{SU}(2)$. yet it turns out, quite remarkably, that all its anomalies vanish over the set of fermions shown in table 1 . This includes both the anomalies of $\mathrm{U}(1)_{b}$ alone and its mixed anomalies with $\mathrm{SU}(5) \times \mathrm{U}(1)_{X} \times \mathrm{U}(1)_{a}$. 


\begin{tabular}{|c|r|rrr|r|r|r|}
\hline & & & & \multicolumn{1}{|c|}{$X=$} & $a=$ & \multicolumn{2}{c|}{$b=$} \\
{$[\mathrm{SU}(6) \times \mathrm{SU}(2)]^{T}$} & $\mathrm{SU}(5)$ & $T_{6}$ & $T_{2}$ & $T$ & $T_{6}+5 T_{2}$ & $2 T_{2}$ & $\frac{2}{3} T_{6}+\frac{1}{3} T$ \\
\hline$(15,1)^{1}$ & 10 & 1 & 0 & 1 & 1 & 0 & 1 \\
$"$ & 5 & -2 & 0 & 1 & -2 & 0 & -1 \\
\hline$(\overline{6}, 2)^{-2}$ & $\overline{5}$ & $-\frac{1}{2}$ & $\frac{1}{2}$ & -2 & 2 & 1 & -1 \\
$"$ & 1 & $\frac{5}{2}$ & $\frac{1}{2}$ & -2 & 5 & 1 & 1 \\
$"$ & $\overline{5}$ & $-\frac{1}{2}$ & $-\frac{1}{2}$ & -2 & -3 & -1 & -1 \\
$"$ & 1 & $\frac{5}{2}$ & $-\frac{1}{2}$ & -2 & 0 & -1 & 1 \\
\hline$(1,3)^{3}$ & 1 & 0 & 1 & 3 & 5 & 2 & 1 \\
$"$ & 1 & 0 & 0 & 3 & 0 & 0 & 1 \\
$"$ & 1 & 0 & -1 & 3 & -5 & -2 & 1 \\
\hline
\end{tabular}

Table 5. How the generators of $\mathrm{U}(1)_{X}, \mathrm{U}(1)_{a}$, and $\mathrm{U}(1)_{b}$ are related to those of $\mathrm{SU}(6) \times \mathrm{SU}(2) \times$ $\mathrm{U}(1)_{T}$.

This involves altogether eight trace conditions: $\operatorname{Tr}\left(b^{3}\right)=0, \operatorname{Tr}(b)=0, \operatorname{Tr}\left(b^{2} X\right)=0$, $\operatorname{Tr}\left(b X^{2}\right)=0, \operatorname{Tr}\left(b^{2} a\right)=0, \operatorname{Tr}\left(b a^{2}\right)=0, \operatorname{Tr}(b a X)=0$, and $\operatorname{Tr}\left(b\left(\lambda_{5}\right)^{2}\right)=0$. Because of this it is possible to gauge the full group $\mathrm{SU}(5) \times \mathrm{U}(1)_{X} \times \mathrm{U}(1)_{a} \times \mathrm{U}(1)_{b}$. However, it is not necessary to gauge $\mathrm{U}(1)_{b}$ to insure proton stability. If $\mathrm{U}(1)_{b}$ is not gauged, and therefore presumably broken explicitly by gravity effects, then several more Yukawa terms would be allowed besides those shown in table 3. (In particular, it would allow the coupling $\overline{5}^{(-3)} \overline{5}^{(2)}\left\langle 10_{H}^{(1)}\right\rangle$.) Those additional terms would cause Standard Model leptons to mix with heavy, vectorlike leptons, but it can easily be shown that it would not cause protons to decay.

Though it is not important for proton stability that $\mathrm{U}(1)_{b}$ be anomaly free, it is quite interesting that it is, since it involves eight independent non-trivial conditions, as we saw. The question is whether there is also some underlying group-theoretical explanation for these cancellations based on embedding in $\mathrm{SU}(6) \times \mathrm{SU}(2)$, as there was for the anomalyfreedom of $\mathrm{U}(1)_{a}$. It happens there is a partial explanation, as we will now see.

Consider the group $\mathrm{SU}(6) \times \mathrm{SU}(2) \times \mathrm{U}(1)_{T}$, where $(15,1)$ has $T=1,(\overline{6}, 2)$ has $T=-2$, and $(1,3)$ has $T=3$. One can easily easily check that $\mathrm{U}(1)_{T}$ is anomaly-free. There are four conditions:

$$
\begin{aligned}
\operatorname{Tr}\left(T^{3}\right) & =15 \cdot(1)^{3}+12 \cdot(-2)^{3}+3 \cdot(3)^{3}=15-96+81=0, \\
\operatorname{Tr}(T) & =15 \cdot(1)+12 \cdot(-2)+3 \cdot(3)=15-24+9=0, \\
\operatorname{Tr}\left(\left(T_{6}\right)^{2} T\right) & =1 \cdot 4 \cdot(1)+2 \cdot 1 \cdot(-2)=0, \\
\operatorname{Tr}\left(\left(T_{2}\right)^{2} T\right) & =6 \cdot 1 \cdot(-2)+1 \cdot 4 \cdot(3)=0 .
\end{aligned}
$$

Though this is a surprising coincidence, it is far less surprising than the satisfying of eight anomaly-cancellation conditions for $\mathrm{U}(1)_{b}$ at the $\mathrm{SU}(5) \times \mathrm{U}(1)_{X}$ level. If one now defines $b \equiv \frac{2}{3} T_{6}+\frac{1}{3} T$, one discovers that the representations in table 1 have exactly the $b$ values given in table 4. All of this is displayed in table 5. As noted above, the group $\mathrm{U}(1)_{a}$ must be local to prevent gravity-induced proton decay. If $\mathrm{U}(1)_{a}$ is gauged, however, its gauge boson could create difficulties. For if $\mathrm{U}(1)_{a}$ is not spontaneously broken there is a new 
long-range force, while if it is spontaneously broken the proof of proton stability could be invalidated, since it depended on conservation of $a$.

First, consider the case that $\mathrm{SU}(5) \times \mathrm{U}(1)_{X} \times \mathrm{U}(1)_{a}$ is not embedded in $\mathrm{SU}(6) \times \mathrm{SU}(2)$. Then one can simply introduce a scalar field $\eta$ which is neutral under $\mathrm{SU}(5) \times \mathrm{U}(1)_{X}$ but has charge $a(\eta) \neq 0$ under $\mathrm{U}(1)_{a}$. This field can obtain a vacuum expectation value that makes the mass of the $\mathrm{U}(1)_{a}$ gauge boson large enough to avoid conflict with experiment. (There is also nothing to prevent the gauge coupling of $\mathrm{U}(1)_{a}$ being small.) The existence of such a field would modify eqs. (1) and (2). One must put into the operator of eq. (1) a factor $(\langle\eta\rangle)^{w}$, where $w$ is some integer. Then an additional term $w a(\eta)$ would appear on the left-hand side of eq. (2). This changes eq. (4) to $B=w a(\eta)$. As long as $a(\eta)$ is not of the form $1 / w$ for some integral value of $w$, proton decay cannot happen.

Another possibility is that $\mathrm{U}(1)_{a}$ is not spontaneously broken, but has such a tiny gauge coupling constant that the resulting long-range force has not been seen. This seems highly implausible, but is certainly possible.

If $\mathrm{SU}(5) \times \mathrm{U}(1)_{X} \times \mathrm{U}(1)_{a}$ is embedded in $\mathrm{SU}(6) \times \mathrm{SU}(2)$, then the possibilities are more limited. The gauge coupling of $\mathrm{U}(1)_{a}$ cannot then be arbitrarily small, and the possible values of $a(\eta)$ are restricted. Moreover, if $\langle\eta\rangle$ is large compared to the electroweak scale, one requires that it break $\mathrm{U}(1)_{a}$ without breaking the electroweak gauge group. The smallest $\mathrm{SU}(6) \times \mathrm{SU}(2)$ multiplet that has a component that can do this is $(6,2)$. Then $\eta^{6,2}$ (where the 6 is the $\mathrm{SU}(6)$ index and the 2 is the $\mathrm{SU}(2)$ index) has $Y / 2=0, I_{2 L}=0$, and $a=1$. But this value of $a(\eta)$ allows the proton to decay. The smallest multiplet that can break $\mathrm{U}(1)_{a}$ above the electroweak without allowing proton decay is a $(21,3)$ of $\mathrm{SU}(6) \times \mathrm{SU}(2)$. This has a component that has $I_{2 L}=Y / 2=0$ and $a=2$. This allows operators that give $\Delta B= \pm 2$, and thus possibly neutron-antineutron oscillations, but not proton decay.

\section{The lepton phenomenology of the model}

The model presented above has six doublets of extra leptons (two for each family). This raises several possible phenomenological problems, including consistency with the measured value of the $\rho$ parameter, the rates for $H \rightarrow \gamma \gamma$ and $H \rightarrow Z^{0} \gamma$, and the stability of the Higgs potential. We shall discuss these in turn.

The effect of the new fermions on the $\rho$ parameter can be made small if the extra lepton doublets are not "split", i.e. if the neutral and charged components have the same or nearly the same mass. This seems somewhat artificial, but perhaps could be enforced by some symmetry, though we have not investigated this possibility.

The extra lepton doublets will definitely contribute very significantly to the amplitude for $H \rightarrow \gamma \gamma$. This process comes, as is well known, from one-loop triangle graphs, where in the Standard Model the amplitude is dominated by the $W$ boson loop and $t$ quark loop [17-20]. In the model presented here, one must include the diagrams with the extra charged leptons running around the loop. The matrix element squared for $H \rightarrow \gamma \gamma$ is given by $|M|^{2}=\frac{g^{2} m_{H}^{4}}{32 \pi^{2} m_{W}^{2}}\left|\sum_{i} \alpha N_{c} e_{i}^{2} F_{i}\right|^{2}$, where $i$ stands for the type of particle in the loop, and $F_{i}$ is given (for $i$ being a gauge boson, fermion, or scalar, respectively) by $F_{\text {gauge }}=$ $2+3 \tau+3 \tau(2-\tau) f(\tau), F_{\text {fermion }}=-2 \tau(1+[1-\tau] f(\tau)), F_{\text {scalar }}=\tau(1-\tau f(\tau))$, for $\tau>1$, 
where $\tau \equiv\left(2 m_{i} / m_{H}\right)^{2}$, and $f(\tau)=\left(\sin ^{-1} \sqrt{1 / \tau}\right)^{2}$. For the Standard Model contributions, one has $F_{S M} \cong F_{W}+F_{t} \cong+8.4-1.8=6.6$. Since the six new charged leptons in our model must be heavy enough not to have been seen, $\tau$ for them is large and $F_{L^{ \pm}} \cong 6(-4 / 3) \cong-8$. This is larger than the Standard Model contribution and of opposite sign. If there are additional fermions that contribute to $H \rightarrow \gamma \gamma$, the total amplitude can be close to -1 times the Standard Model value, giving the same rate. Or, depending on the number and type of additional fermions, the rate could be somewhat smaller or larger than the Standard Model prediction.

The process $H \rightarrow Z^{0} \gamma[20,21]$ is not a difficulty for the model. The present limits on this decay are very loose, and the contribution of charged leptons to it are highly suppressed, since the $Z$ coupling to the charged leptons is proportional to the well-known factor $I_{3 L}-2 Q \sin ^{2} \theta_{W}=-\frac{1}{2}+2(0.23) \cong-0.04$.

The presence of six lepton doublets with $O(1)$ Yukawa couplings will give large radiative contributions to the Higgs quartic self-coupling. However, we envision the unification scale being much lower than it is in typical unified models, and the unified theory may be effective theory valid only below some cutoff. If that cutoff scale is relatively low, the Higgs quartic coupling can remain positive below that scale.

We conclude that the existence of the extra leptons is compatible with present limits. One might worry, on the other hand, that the new quarks $D=D^{c}$ would present phenomenological problems, for instance by substantially affecting the $H \rightarrow 2$ gluon amplitude. However, a curious feature of our model is that the extra $D+D^{c}$ quarks (unlike the extra leptons) do not couple to the Standard Model Higgs doublet, but get their mass from a Standard Model singlet Higgs field $\left(\Omega^{12}\right)$. Thus they do not contribute to the Higgs decay amplitudes, the $\rho$ parameter, or the running of the Higgs quartic coupling. Moreover, their mass could be much higher than the weak scale.

Returning to the leptons, there remains the question of neutrino mass. Realistic neutrino masses seem at first sight to be a problem for the model. The first question is how those masses can be fractions of an $\mathrm{eV}$, since this would not emerge from the usual seesaw mechanisms if the unification scale is very low. The second question is how to avoid neutrino masses that are of the same order as the quark and charged lepton masses.

If one looks at the Yukawa couplings allowed by $\mathrm{SU}(5) \times \mathrm{U}(1)_{X} \times \mathrm{U}(1)_{a} \times \mathrm{U}(1)_{b}$, all of which are shown in table 3 , one finds several mass terms for neutral fermions. Specifically, the second, eighth, and tenth lines of table 3 have operators that give, respectively, operators of the form $\nu^{c} \nu\left\langle H^{*}\right\rangle, N^{\prime \prime} \bar{N}^{\prime \prime}\langle H\rangle$, and $\nu N^{\prime \prime}\langle\tilde{H}\rangle$. Ignoring family indices, this gives a mass matrix of the following form:

$$
\left(\nu, \nu^{c}, N^{\prime \prime}, \bar{N}^{\prime \prime}\right)\left(\begin{array}{cccc}
0 & \left\langle H^{*}\right\rangle & \langle\tilde{H}\rangle & 0 \\
\left\langle H^{*}\right\rangle & 0 & 0 & 0 \\
\langle\tilde{H}\rangle & 0 & 0 & \langle H\rangle \\
0 & 0 & \langle H\rangle & 0
\end{array}\right)\left(\begin{array}{c}
\nu \\
\nu^{c} \\
N^{\prime \prime} \\
\bar{N}^{\prime \prime}
\end{array}\right)
$$

This matrix has non-zero determinant, and the VEVs that appear in it are of order the electroweak scale. Thus, one would not expect neutrino masses of order a fraction of an $\mathrm{eV}$ unless some Yukawa couplings were extremely small. For example, if the Yukawa coupling 
in the terms $\nu^{c} \nu\left\langle H^{*}\right\rangle$, and $N^{\prime \prime} \bar{N}^{\prime \prime}\langle H\rangle$ were of order $\epsilon$ and those in $\nu N^{\prime \prime}\langle\tilde{H}\rangle$ were of order 1 , then there would be (for each family) one pseudo-Dirac neutrino with mass of order the electroweak scale (composed approximately of $\nu$ and $N^{\prime \prime}$ ) and one pseudo-Dirac neutrino with mass of order $\epsilon^{2}$ times the electroweak scale (composed approximately of $\nu^{c}$ and $\bar{N}^{\prime \prime}$ ), as can be seen from the form of eq. (5). One would therefore need to have $\epsilon$ be of order $10^{-6}$ to $10^{-7}$ even for the third family. This seems contrived.

A more attractive possibility arises if there is an additional type of neutral fermion introduced for each family. Let is call it $S$ and say that it is neutral under $\operatorname{SU}(5) \times$ $\mathrm{U}(1)_{X} \times \mathrm{U}(1)_{a}$, but has $b=-1$. Then one can have a coupling of the type $\psi^{12} S\left\langle\left(\Omega^{12}\right)^{*}\right\rangle=$ $\nu^{c} S\left\langle\left(\Omega^{12}\right)^{*}\right\rangle$, which is contained in $10^{(1)} 1^{(0)}\left\langle\left(10_{H}^{(1)}\right)^{*}\right\rangle$. This term is invariant under $\mathrm{SU}(5) \times$ $\mathrm{U}(1)_{X} \times \mathrm{U}(1)_{a} \times \mathrm{U}(1)_{b}$. The mass matrix then has the form

$$
\left(S, \nu, \nu^{c}, N^{\prime \prime}, \bar{N}^{\prime \prime}\right)\left(\begin{array}{ccccc}
0 & 0 & \langle\Omega\rangle & 0 & 0 \\
0 & 0 & \left\langle H^{*}\right\rangle & \langle\tilde{H}\rangle & 0 \\
\langle\Omega\rangle & \left\langle H^{*}\right\rangle & 0 & 0 & 0 \\
0 & \langle\tilde{H}\rangle & 0 & 0 & \langle H\rangle \\
0 & 0 & 0 & \langle H\rangle & 0
\end{array}\right)\left(\begin{array}{c}
S \\
\nu \\
\nu^{c} \\
N^{\prime \prime} \\
\bar{N}^{\prime \prime}
\end{array}\right) .
$$

This matrix has one zero eigenvalue. So (for each family) there is a massless neutral fermion. These can be given tiny masses in various ways, one of which we will describe shortly. These light neutrinos are linear combinations of $S, \nu$ and $\bar{N}^{\prime \prime}$, as can be seen from eq. (6). Of course, to be consistent with bounds on lepton universality, these linear combinations would have to be mostly $\nu$; but this only requires certain ratios of Yukawa couplings in eq. (6) to be of order $10^{-1}$.

One can give a small mass to the neutrinos by a higher-dimension operator of the form $S S\langle\zeta\rangle^{n}$, where $\zeta$ is a $1^{(0)}$ of $\mathrm{SU}(5) \times \mathrm{U}(1)_{X}$ and has $a=0$ and $b=1 / n$. Such an operator might be induced by gravity. The value of $n$ needed to get realistic neutrino masses would depend on the gravity scale.

\section{Low scale grand unification?}

If a unified model has an absolutely stable proton due to an exact symmetry, then obviously proton lifetime limits would not constrain the unification scale at all. The question would then arise how low the unification scale could be. Could it be near the electroweak scale? If the unification scale is low, one has to explain how the gauge couplings are able to unify. One possibility is to exploit an idea first proposed by Shafi and Wetterich many years ago [22] (see also [23, 24]).

Let us consider an effective operator for physics below some cutoff scale $\Lambda$ given by

$$
\frac{c}{\Lambda} \operatorname{Tr}\left(G_{\mu \nu} G^{\mu \nu} \mathcal{A}\right),
$$

where $G_{\mu \nu}$ is the Grand Unified Theory field strength and $\mathcal{A}$ is a scalar multiplet in the adjoint representation of $\mathrm{SU}(5)$. The scale $\Lambda$ is kept as a free parameter for the time being. Upon symmetry breaking at the unification scale $M_{U}$, the Higgs field gets a vacuum 
expectation value $\langle\mathcal{A}\rangle=M_{U}(2,2,2,-3,-3) / \sqrt{50 \pi \alpha_{G}}$, where $\alpha_{G}$ is the value of the $\mathrm{SU}(5)$ gauge coupling at $M_{U}$.

The dimension 5 operator modifies the gauge kinetic terms of $\mathrm{SU}(3) \times \mathrm{SU}(2) \times \mathrm{U}(1)$ below the scale $M_{u}$ to

$$
-\frac{1}{4}\left(1+\epsilon_{1}\right) F_{\mu \nu} F_{\mathrm{U}(1)}^{\mu \nu}-\frac{1}{2}\left(1+\epsilon_{2}\right) \operatorname{Tr}\left(F_{\mu \nu} F_{\mathrm{SU}(2)}^{\mu \nu}\right)-\frac{1}{2}\left(1+\epsilon_{3}\right) \operatorname{Tr}\left(F_{\mu \nu} F_{\mathrm{SU}(3)}^{\mu \nu}\right)
$$

with

$$
\epsilon_{1}=\frac{\epsilon_{2}}{3}=-\frac{\epsilon_{3}}{2}=\frac{\sqrt{2}}{5 \sqrt{\pi}} \frac{c}{\sqrt{\alpha_{G}}} \frac{M_{u}}{\Lambda} .
$$

If we were to take $\Lambda=M_{u}$, then

$$
\epsilon_{1}=\frac{\epsilon_{2}}{3}=-\frac{\epsilon_{3}}{2}=\frac{\sqrt{2}}{5 \sqrt{\pi}} \frac{c}{\sqrt{\alpha_{G}}}
$$

We can now perform a finite field redefinition $A_{\mu}^{i} \rightarrow\left(1+\epsilon_{i}\right)^{1 / 2} A_{\mu}^{i}$ to canonically normalize the kinetic terms of the gauge bosons. Then the corresponding redefined coupling constants are $g_{i} \rightarrow\left(1+\epsilon_{i}\right)^{-1 / 2} g_{i}$. We get the unification condition:

$$
\alpha_{G}=\left(1+\epsilon_{1}\right) \alpha_{1}\left(M_{u}\right)=\left(1+\epsilon_{2}\right) \alpha_{2}\left(M_{u}\right)=\left(1+\epsilon_{3}\right) \alpha_{3}\left(M_{u}\right) .
$$

We now wish to consider low scale unification. Direct observational bounds on the heavy gauge bosons of $\mathrm{SU}(5) / G_{S M}$ as well as on the color octet scalars lead us to consider a unification scale in the few $\mathrm{TeV}$ region. We thus take $M_{U}$ a few $\mathrm{TeV}$ which implies that there is very little running for the gauge couplings and we can use the LEP values, at least to first approximation. We take $\alpha_{2}\left(M_{Z}\right)=0.03322$ and $\alpha_{3}\left(M_{Z}\right)=0.118$. Since $\alpha_{1}$ is a free parameter, we will use $c$ to obtain the numerical unification of $\alpha_{2}$ and $\alpha_{3}$. We need

$$
c=5 \sqrt{\frac{\pi}{2}}\left(\frac{\alpha_{2}-\alpha_{3}}{3 \alpha_{2}+2 \alpha_{3}}\right) \sqrt{\alpha_{G}} .
$$

We then find

$$
\epsilon_{1}=5\left(\frac{\alpha_{2}-\alpha_{3}}{3 \alpha_{2}+2 \alpha_{3}}\right) \frac{1}{\alpha_{G}}
$$

and thus

$$
\alpha_{1}=\frac{\alpha_{G}}{1+5\left(\frac{\alpha_{2}-\alpha_{3}}{3 \alpha_{2}+2 \alpha_{3}}\right) \frac{1}{\alpha_{G}}}
$$

Numerically we have $c=-1.58 / \sqrt{\alpha_{G}}$. If I take $\alpha_{G}=0.05$ for illustration, We get $c=-7$. In a sense we see that if the grand unified theory is strongly coupled, the Wilsonian expansion works best as the Wilson coefficients get smaller: for $\alpha_{G}=1$, we get $c=-1.58$.

The $\mathrm{U}(1)$ of hypercharge is not purely a subgroup of $\mathrm{SU}(5)$, but lies partly in the $\mathrm{U}(1)_{X}$, whose gauge coupling is an independent, free parameter. This coupling can be chosen to give the observed value of the hypercharge (and electromagnetic) gauge coupling.

Interestingly, the Planck scale could also be lowered to the TeV region to remove all hierarchies. There are two known mechanisms for that. One is to assume that large extra 
dimensions open up at an energy scale of a few $\mathrm{TeV}[25-27]$. The other one relies on a large hidden sector of particles which lead to a running of the Planck mass [28]. Note that the running can also be obtained by a scalar field with a large non-minimal coupling to the Ricci scalar [29]. As pointed out in [30, 31], large extra dimensions are a natural framework to incorporate low scale unification.

\section{Acknowledgments}

We thank K.S. Babu, Ilia Gogoladze, and Qaisar Shafi for useful conversations. This work is supported in part by DOE grant No. DE-FG02-12ER41808, by the European Cooperation in Science and Technology (COST) action MP0905 "Black Holes in a Violent Universe" and by the Science and Technology Facilities Council (grant number ST/J000477/1).

Open Access. This article is distributed under the terms of the Creative Commons Attribution License (CC-BY 4.0), which permits any use, distribution and reproduction in any medium, provided the original author(s) and source are credited.

\section{References}

[1] J.C. Pati and A. Salam, Lepton Number as the Fourth Color, Phys. Rev. D 10 (1974) 275 [Erratum ibid. D 11 (1975) 703] [inSPIRE].

[2] P. Fileviez Perez and M.B. Wise, Low Scale Quark-Lepton Unification, Phys. Rev. D 88 (2013) 057703 [arXiv: 1307.6213] [INSPIRE].

[3] S.M. Barr, Some Comments on Flipped $\mathrm{SU}(5) \times \mathrm{U}(1)$ and Flipped Unification in General, Phys. Rev. D 40 (1989) 2457 [InSPIRE].

[4] A. De Rujula, H. Georgi and S.L. Glashow, Flavor goniometry by proton decay, Phys. Rev. Lett. 45 (1980) 413 [INSPIRE].

[5] H. Georgi, S.L. Glashow and M. Machacek, $\mu^{+}$Polarization in Proton Decay: A Probe of Flavor Mixing in Unified Models, Phys. Rev. D 23 (1981) 783 [InSPIRE].

[6] S.M. Barr, A New Symmetry Breaking Pattern for $\mathrm{SO}(10)$ and Proton Decay, Phys. Lett. B 112 (1982) 219 [INSPIRE].

[7] C. Jarlskog, Inherent Mixings in the Grand Unified SU(5) Model and Proton Lifetime, Phys. Lett. B 82 (1979) 401 [INSPIRE].

[8] R.N. Mohapatra, Flavor Mixing in SU(5) Grand Unified Theories, Phys. Rev. Lett. 43 (1979) 893 [INSPIRE].

[9] S. Nandi, A. Stern and E.C.G. Sudarshan, Can proton decay be rotated away?, Phys. Lett. B 113 (1982) 165 [INSPIRE].

[10] I. Dorsner and P. Fileviez Perez, Could we rotate proton decay away?, Phys. Lett. B 606 (2005) 367 [hep-ph/0409190] [INSPIRE].

[11] G. Segre and H.A. Weldon, SU(5) Theories With Both Proton Stability and Cosmological Baryon Number Generation, Phys. Rev. Lett. 44 (1980) 1737 [INSPIRE].

[12] Z. Berezhiani, I. Gogoladze and A. Kobakhidze, TeV scale unification in four-dimensions versus extra dimensions, Phys. Lett. B 522 (2001) 107 [hep-ph/0104288] [INSPIRE]. 
[13] A.B. Kobakhidze, Proton stability in TeV scale GUTs, Phys. Lett. B 514 (2001) 131 [hep-ph/0102323] [INSPIRE].

[14] S.M. Barr, Rotating Away Proton Decay in Flipped Unification, Phys. Rev. D 88 (2013) 057702 [arXiv: 1307.5770] [INSPIRE].

[15] P. Fileviez Perez and M.B. Wise, Breaking Local Baryon and Lepton Number at the TeV Scale, JHEP 08 (2011) 068 [arXiv: 1106.0343] [INSPIRE].

[16] M. Duerr, P. Fileviez Perez and M.B. Wise, Gauge Theory for Baryon and Lepton Numbers with Leptoquarks, Phys. Rev. Lett. 110 (2013) 231801 [arXiv:1304.0576] [INSPIRE].

[17] J.R. Ellis, M.K. Gaillard and D.V. Nanopoulos, A Phenomenological Profile of the Higgs Boson, Nucl. Phys. B 106 (1976) 292 [inSPIRE].

[18] M.A. Shifman, A.I. Vainshtein, M.B. Voloshin and V.I. Zakharov, Low-Energy Theorems for Higgs Boson Couplings to Photons, Sov. J. Nucl. Phys. 30 (1979) 711 [InSPIRE].

[19] L. Bergstrom, G. Hulth and H. Snellman, Relativistic Calculation of the Two Photon Coupling of Scalar and Tensor Mesons, Z. Phys. C 16 (1983) 263 [inSPIRE].

[20] J. Gunion, H. Haber, G. Kane and S. Dawson, The Higgs Hunter's Guide, Addison-Wesley, Reading (1990).

[21] R.N. Cahn, M.S. Chanowitz and N. Fleishon, Higgs Particle Production by $Z \rightarrow H \gamma$, Phys. Lett. B 82 (1979) 113 [INSPIRE].

[22] Q. Shafi and C. Wetterich, Modification of GUT Predictions in the Presence of Spontaneous Compactification, Phys. Rev. Lett. 52 (1984) 875 [INSPIRE].

[23] L.J. Hall and U. Sarid, Gravitational smearing of minimal supersymmetric unification predictions, Phys. Rev. Lett. 70 (1993) 2673 [hep-ph/9210240] [INSPIRE].

[24] X. Calmet, S.D.H. Hsu and D. Reeb, Grand unification and enhanced quantum gravitational effects, Phys. Rev. Lett. 101 (2008) 171802 [arXiv:0805. 0145] [INSPIRE].

[25] N. Arkani-Hamed, S. Dimopoulos and G.R. Dvali, The Hierarchy problem and new dimensions at a millimeter, Phys. Lett. B 429 (1998) 263 [hep-ph/9803315] [INSPIRE].

[26] I. Antoniadis, N. Arkani-Hamed, S. Dimopoulos and G.R. Dvali, New dimensions at a millimeter to a Fermi and superstrings at a TeV, Phys. Lett. B 436 (1998) 257 [hep-ph/9804398] [INSPIRE].

[27] L. Randall and R. Sundrum, A Large mass hierarchy from a small extra dimension, Phys. Rev. Lett. 83 (1999) 3370 [hep-ph/9905221] [INSPIRE].

[28] X. Calmet, S.D.H. Hsu and D. Reeb, Quantum gravity at a TeV and the renormalization of Newton's constant, Phys. Rev. D 77 (2008) 125015 [arXiv:0803.1836] [inSPIRE].

[29] M. Atkins and X. Calmet, Unitarity bounds on low scale quantum gravity, Eur. Phys. J. C 70 (2010) 381 [arXiv: 1005.1075] [INSPIRE].

[30] K.R. Dienes, E. Dudas and T. Gherghetta, Extra space-time dimensions and unification, Phys. Lett. B 436 (1998) 55 [hep-ph/9803466] [INSPIRE].

[31] K.R. Dienes, E. Dudas and T. Gherghetta, Grand unification at intermediate mass scales through extra dimensions, Nucl. Phys. B 537 (1999) 47 [hep-ph/9806292] [INSPIRE]. 